\title{
ÁCIDOS GRASOS OMEGA-3 PARA LA PREVENCIÓN Y TRATAMIENTO DE LAS DEPRESIONES EN EL EMBARAZO Y POST PARTO
}

\author{
Alexis Tapia S. $^{1}$ \\ ${ }^{1}$ Instituto Nacional de Tecnología de los Alimentos (INTA), Laboratorio de Gastroenterología, Universidad de Chile.
}

\section{RESUMEN}

Las depresiones que se presentan durante el embarazo y después del parto constituyen un importante problema de salud pública. Si bien la etiología es desconocida, su aparición se asocia a la depleción de ácidos grasos omega-3. Estado propio del embarazo, pues la madre traspasa sus reservas al feto, para la formación del cerebro de éste. Consecuentemente, la suplementación con ácidos grasos omega-3 ha demostrado ser eficaz para tratar la depresión tanto en embarazadas como en otro tipo de pacientes. Por lo tanto, es aconsejable suplementar a las embarazadas con estos ácidos grasos para prevenir y tratar las depresiones relacionadas al embarazo. Esta acción tiene las ventajas de ser inocuo durante el embarazo y la lactancia, además de traspasarse al feto durante estos períodos, permitiendo su adecuado desarrollo cerebral.

\section{PALABRAS CLAVES: Embarazo, depresión post parto, ácidos grasos omega-3}

\section{SUMMARY}

The depressions that appear during the pregnancy and post partum constitute an important problem of public health. Although the etiology is not known, its appearance is associated to the depletion of omega-3 fatty acids. Own state of the pregnancy, because the mother transfers her reserves to the fetus, for the formation of his brain. Consequently the supplementation with omega-3 fatty acids, has demonstrated to be effective to treat the depression as much in pregnants as another type of patients. Therefore, it is advisable the supplementation to the pregnant women with these fatty acids to prevent and to treat the depressions related to the pregnancy. This action has the advantages of to be innocuous during the pregnancy and in lactation, besides to transfer to the fetus during these periods, allowing his adequate cerebral development.

\section{KEY WORDS: Pregnancy, post partum depression, omega-3 fatty acids}

\section{INTRODUCCIÓN}

La depresión post parto es un importante problema de salud pública, ya que dicho trastorno ha demostrado ser muy frecuente en distintas series. Como ejemplo, en Chile de 106 mujeres estudiadas, el $32,1 \%$ presentó depresión según los criterios diagnósticos de la décima revisión de la Clasificación Internacional de Enfermedades (CIE-10) (1). La aparición de depresión no sólo afecta a mujeres después del parto, sino que también a la mismas embarazadas, como lo comprobó un estudio de Brasil en el cual de 120 adolescentes embarazadas, el $37,5 \%$ presentó síntomas de depresión, evaluadas mediante la Escala Hospitalaria de Ansiedad y Depresión (HAD) 
y la Escala de Ideación Suicida de Beck (2). Por lo tanto, es necesario desarrollar nuevas estrategias para prevenir su aparición y tratarla efectivamente una vez que se manifiesta.

A pesar de que la etiología de la depresión aún es desconocida, existen determinados factores que se asocian a su aparición y remisión. En la población general se ha observado que pacientes afectados por episodios de depresión mayor poseen disminuidos niveles de ácidos grasos omega-3 en fosfolípidos plasmáticos, ésteres de colesterol, en membranas de eritrocitos y en tejido adiposo (3-7). También se han asociado regiones con bajo consumo de alimentos que aportan estos ácidos grasos, con elevadas prevalencias de depresión (8). En forma concordante, se han realizado intervenciones en pacientes depresivos, que consisten en suplementarlos con ácidos grasos omega-3, por cortos períodos, lográndose mejoras estadísticamente significativas de los síntomas depresivos $(9,10)$, incluso de la ideación suicida en pacientes resistentes a tratamiento (11).

La asociación de los ácidos grasos omega-3 con la depresión puede ser fundamental en la embarazadas, ya que ellas traspasan sus reservas al feto, lo que puede ocasionar una depleción de los mismos en la madre. En este trabajo se analizarán la importancia de los ácidos grasos omega-3 en el desarrollo cerebral, funcionamiento cerebral y su relación con la depresión durante el embarazo y post parto.

\section{ÁCIDOS GRASOS OMEGA-3}

Los ácidos grasos poliinsaturados linoleico (AL: 18:2n6) y $\alpha$-linolénico (AAL: 18:3n3) son los precursores de las familias omega- 6 y omega-3, respectivamente. Estos ácidos grasos AL y AAL no pueden ser sintetizados por los humanos, por lo tanto la dieta es la única fuente de estos. Por este motivo son considerados esenciales. El AAL es aportado principalmente por el consumo de pescado, aceite de canola, aceite de soja y nueces. El $A L$ se encuentra en aceites vegetales, margarinas, carnes magras y huevos.

Los principales derivados en la vía omega-3 son los ácidos eicosapentaenoico (EPA 20:5 n-3) y docosahexaenoico (DHA 22:6 n-3). En la vía omega-6 el más importante es el ácido araquidónico (AA 20:4 n-6). A diferencia de otras especies animales los humanos poseen una limitada capacidad para producir EPA y DHA, lo que ocasiona que la principal fuente de estos sea la alimentaria (12).
Importancia de los ácidos grasos omega-3 para la formación y funcionamiento del sistema nervioso central. El desarrollo del sistema nervioso central (SNC), particularmente del cerebro, se lleva a cabo en el humano durante el último trimestre del embarazo. Es en este período donde comienza en forma activa la formación de las neuronas y donde el requerimiento de DHA aumenta considerablemente (13). En efecto, el cerebro de los primates acumula este ácido en la vida intrauterina y durante el primer año de vida $(14,15)$. En el útero, el DHA es aportado desde las reservas de la madre. Esto produce que la concentración de DHA en el cerebro (donde llega a constituir el $40 \%$ del contenido de ácidos grasos poliinsaturados de cadena larga) es mayor que la concentración en el plasma fetal y ésta, a su vez, mayor que la de la placenta y del plasma materno. La barrera hematoencefálica es impermeable a los ácidos grasos saturados monoinsaturados y al colesterol, los cuales deben ser formados por el cerebro, en cambio es permeable a los ácidos grasos omega-6 y omega-3, permitiendo así su aporte externo. En las etapas tardías del último trimestre gestacional, los astrocitos adquieren la función de suplir con DHA a las neuronas en formación. Los ácidos grasos omega-3 también son esenciales para el tejido visual, estructura derivada del sistema nervioso central, que al igual que el cerebro tiene una extraordinaria capacidad para captar DHA desde el plasma. En la retina, el DHA forma parte de los fotorreceptores de los conos y bastoncitos. Estas estructuras de la membrana, asociadas a la rodopsina, participan en la conversión del estímulo luminoso en uno eléctrico y en los procesos de transducción de señales que acompañan a este fenómeno. No hay evidencias que la retina pueda sintetizar DHA a partir de sus precursores. Sin embargo, este ácido graso es continuamente reutilizado en el tejido, ya que el recambio de los conos y de los bastoncitos es muy activo (13).

Un hecho que aclara que los ácidos grasos esenciales juegan un rol activo en el funcionamiento de las membranas neuronales, es que ellos corresponden al $45 \%$ de los ácidos grasos presentes en las membranas sinápticas, por lo mismo se considera que los ácidos grasos poliinsaturados y el colesterol, son los principales determinantes de las propiedades biofísicas de las membranas neuronales (12).

Como ejemplo de la importancia de los ácidos grasos poliinsaturados de cadena larga en la constitución del sistema nerviosos central, se 
puede considerar que el cerebro contiene una alta concentración de estos ácidos, que corresponde a alrededor del $20 \%$ de su peso seco y en el sistema nervioso central uno de cada tres ácidos grasos es poliinsaturado $(16,17)$.

Como corolario es importante destacar que, en animales, se ha demostrado que dietas deficientes en ácidos grasos omega-3 modifican la composición de lípidos y funciones neuroquímicas en áreas específicas del cerebro (18), además se ha evidenciado un aumento en la densidad de receptores $2 \mathrm{~A}$ de serotonina en la corteza frontal y una disminución de los receptores D2 de dopamina (19). Por lo tanto, dietas carentes en ácidos grasos omega-3 realmente modifican el funcionamiento cerebral.

Para que los ácidos grasos omega-3 cumplan sus funciones en el SNC, no sólo es importante ingerirlos, sino que hacerlo en las cantidades adecuadas, ya que está demostrado que los niveles de ácidos grasos específicos en el plasma son reflejo de los ácidos grasos consumidos en la dieta (20-22). Del mismo modo la composición de ácidos grasos de los fosfolípidos que circulan en el plasma, se relaciona próximamente a la composición de los fosfolípidos presentes en las membranas de eritrocitos y plaquetas (23).

Además, se ha observado que una dieta alta en ácidos grasos omega-3 aumenta la concentración de los mismos en plasma y membrana de eritrocitos. Es así como el consumo de pescado, aceite de pescado y aceite con DHA produce un aumento de ácidos omega-3 y una disminución de omega- 6 en la fracción lipídica del plasma y en las membranas de eritrocitos y plaquetas (24).

Ácidos grasos omega-3 y depresión en embarazo y post parto. Se ha hipotetizado, en base a estudios observacionales, que la causa de la depresión post parto sería una consecuencia de la depleción de DHA en la madre, pues tiene que transferirle gran cantidad de este ácido graso al feto para la formación del cerebro, por lo tanto este proceso fisiológico produce una depleción de DHA en la madre $(25,26)$.

Un estudio retrospectivo evaluó la presencia de depresión en 112 mujeres mediante la aplicación del Edinburgh Postnatal Depression Scale. Además se les midió DHA en los fosfolípidos plasmáticos y el ácido graso omega-6 docosapentanoico (DPA). Se demostró que las pacientes depresivas presentaron una disminución significativa de la razón DHA/DPA (27). Lo que se produce por una disminución efectiva de los ácidos grasos omega-3.
En otro estudio se incluyeron 48 mujeres embarazadas y se las siguió después del parto, entrevistándolas tres meses después del evento para diagnosticar aparición de depresión. Se utilizaron los criterios del Diagnostic and Statistical Manual of Mental Disorders - Fourth Edition (DSMIV). 10 mujeres desarrollaron depresión post-parto. En comparación a los controles presentaron una disminución de DHA y aumento de las razones omega-6/omega-3 y AA/EPA (28).

Una cohorte de 380 mujeres fue evaluada 6 meses postparto mediante Edinburgh Post-partum Depression Scale y se les midió el DHA plasmático. Un análisis de regresión logística indicó que un aumento de un $1 \%$ de DHA plasmático se asoció con una reducción del $59 \%$ en el reporte de síntomas depresivos (29).

En 2003, se reportó por primera vez el tratamiento de un episodio de depresión mayor en una mujer embarazada de 34 años y cursando la semana 28, esta se negaba a tomar antidepresivos por el temor a la teratogenia, se le administraron 4 g de etil-EPA y $2 \mathrm{~g}$ de DHA al día; mostró mejoría en el ánimo depresivo, en el sentimiento de poco valor, desesperanza y culpa a la semana 4 y desaparición de ideación suicida a la semana 6 , el examen físico del recién nacido fue normal (30).

\section{CONCLUSIONES}

Durante el embarazo las madres pueden llegar a un estado de depleción de ácidos grasos omega3 debido a que traspasan sus reservas para el desarrollo del cerebro fetal. Este estado de depleción se asocia a la aparición de depresión. Este hecho es muy importante, porque puede ser un factor que contribuya a la aparición de depresión durante el embarazo y después del parto. Por lo tanto, es conveniente suplementar a las embarazadas con estos ácidos grasos, ya sea con cápsulas, jarabes o fomentando el consumo de alimentos que los contengan. La suplementación puede ser útil para prevenir la aparición de la depresión y como coadyuvante terapéutico una vez que ella se manifiesta. Además presenta las ventajas de que puede ser administrada durante el embarazo, durante la lactancia, es bien tolerada oralmente, no presenta reacciones adversas ni efectos secundarios. Adicionalmente a sus efectos antidepresivos, es traspasado de la madre al feto durante la gestación y a través de la leche materna, favoreciendo el desarrollo del SNC del recién nacido. 


\section{BIBLIOGRAFÍA}

1. Evans G, Vicuña M, Marín R. Depresión postparto realidad en el sistema público de atención de salud. Rev Chil Obstet Ginecol 2003; 68(6): 491-494.

2. Vas Scavacini G, Botega N. Gravidezvidez Na Adolescência: Prevalência De Depressão, Ansiedade E Ideação Suicida. Rev Assoc Med Bras 2002; 48(3): 245-9.

3. Frasure-Smith $N$, Lesperance $F$, Julien P. Major depression is associated with lower omega-3 fatty acid levels in patients with recent acute coronary syndromes. Biol Psychiatry 2004; 55(9): 891-6.

4. Maes M, Smith R, Christophe A, Cosyns P, Desnyder $\mathrm{R}$, Meltzer $\mathrm{H}$. Fatty acid composition in major depression: decreased omega 3 fractions in cholesteryl esters and increased C20: 4 omega 6/ C20:5 omega 3 ratio in cholesteryl esters and phospholipids. J Affect Disord 1996; 38(1): 35-46.

5. Edwards R, Peet M, Shay J, Horrobin D. Omega-3 polyunsaturated fatty acid levels in the diet and in red blood cell membranes of depressed patients. J Affect Disord 1998; 48(2-3): 149-55.

6. Peet M, Murphy B, Shay J, Horrobin D. Depletion of omega-3 fatty acid levels in red blood cell membranes of depressive patients. Biol Psychiatry 1998; 43(5): 315-9.

7. Mamalakis G, Kiriakakis M, Tsibinos G, Kafatos A. Depression and adipose polyunsaturated fatty acids in the survivors of the Seven Countries Study population of Crete. Prostaglandins Leukot Essent Fatty Acids 2004; 70(6): 495-501.

8. Peet M. International variations in the outcome of schizophrenia and the prevalence of depression in relation to national dietary practices: an ecological analysis. Br J Psychiatry 2004; 184: 404-8.

9. Nemets B, Stahl Z, Belmaker RH. Addition of omega3 fatty acid to maintenance medication treatment for recurrent unipolar depressive disorder. Am J Psychiatry 2002; 159(3): 477-9.

10. Su KP, Huang SY, Chiu CC, Shen WW. Omega-3 fatty acids in major depressive disorder. A preliminary double-blind, placebo-controlled trial. Eur Neuropsychopharmacol 2003; 13(4): 267-71.

11. Puri BK, Counsell SJ, Hamilton G, Richardson AJ, Horrobin DF. Eicosapentaenoic acid in treatmentresistant depression associated with symptom remission, structural brain changes and reduced neuronal phospholipid turnover. Int J Clin Pract 2001; 55(8): 560-3.

12. Bruinsma KA, Taren DL. Dieting, essential fatty acid intake, and depression. Nutr Rev 2000; 58(4): 98-108.

13. Valenzuela A, Nieto MS. Docosahexaenoic acid $(\mathrm{DHA})$ in fetal development and in infant nutrition. Rev Méd Chil 2001; 129(10): 1203-11.

14. Clandinin MT, Chappell JE, Leong S, Heim T, Swyer $\mathrm{PR}$, Chance GW. Intrauterine fatty acid accretion rates in human brain: implications for fatty acid requirements. Early Hum Dev 1980; 4(2): 121-9.
15. Clandinin MT, Chappell JE, Leong S, Heim T, Swyer $\mathrm{PR}$, Chance GW. Extrauterine fatty acid accretion in infant brain: implications for fatty acid requirements. Early Hum Dev 1980; 4(2): 131-8.

16. Bourre JM, Dumont O, Piciotti M, Clement M, Chaudiere J, Bonneil M y cols. Essentiality of omega 3 fatty acids for brain structure and function. World Rev Nutr Diet 1991; 66: 103-17.

17. Yehuda S, Rabinovitz S, Mostofsky DI. Essential fatty acids are mediators of brain biochemistry and cognitive functions. J Neurosci Res 1999; 56: 565570.

18. Delion S, Chalon S, Guilloteau D, Lejeune B, Besnard JC, Durand G. Age-related changes in phospholipid fatty acid composition and monoaminergic neurotransmission in the hippocampus of rats fed a balanced or an n-3 polyunsaturated fatty acid-deficient diet. J Lipid Res 1997; 38(4): 680-9.

19. Delion S, Chalon S, Guilloteau D, Besnard JC, Durand G. Alpha-Linolenic acid dietary deficiency alters age-related changes of dopaminergic and serotoninergic neurotransmission in the rat frontal cortex. J Neurochem 1996; 66(4): 1582-91.

20. Dougherty RM, Galli C, Ferro-Luzzi A, lacono JM. Lipid and phospholipid fatty acid composition of plasma, red blood cells, and platelets and how they are affected by dietary lipids: a study of normal subjects from Italy, Finland, and the USA. Am J Clin Nutr 1987; 45(2): 443-55.

21. Judd JT, Marshall MW, Dupont J. Relationship of dietary fat to plasma fatty acids, blood pressure, and urinary eicosanoids in adult men. J Am Coll Nutr 1989; 8(5): 386-99.

22. Matorras R, Perteagudo L, Sanjurjo P. Biochemical markers of $n-3$ long chain polyunsaturated fatty acid intake during pregnancy. Clin Exp Obstet Gynecol 1998; 25(4): 135-8.

23. Holman RT. Control of polyunsaturated acids in tissue lipids. J Am Coll Nutr 1986; 5: 183-211.

24. Vidgren HM, Agren JJ, Schwab U, Rissanen T, Hanninen O, Uusitupa MI. Incorporation of n-3 fatty acids into plasma lipid fractions, and erythrocyte membranes and platelets during dietary supplementation with fish, fish oil, and docosahexaenoic acid-rich oil among healthy young men. Lipids 1997; 32(7): 697-705.

25. Al MD, van Houwelingen $A C$, Kester $A D$, Hasaart $\mathrm{TH}$, de Jong AE, Hornstra G. Maternal essential fatty acid patterns during normal pregnancy and their relationship to the neonatal essential fatty acid status. Br J Nutr 1995; 74(1): 55-68.

26. Holman RT, Johnson SB, Ogburn PL. Deficiency of essential fatty acids and membrane fluidity during pregnancy and lactation. Proc Natl Acad Sci USA 1991; 88(11): 4835-9.

27. Otto SJ, de Groot RH, Hornstra G. Increased risk of postpartum depressive symptoms is associated with slower normalization after pregnancy of the functional 
docosahexaenoic acid status. Prostaglandins Leukot Essent Fatty Acids 2003; 69(4): 237-43.

28. De Vriese SR, Christophe AB, Maes M. Lowered serum n-3 polyunsaturated fatty acid (PUFA) levels predict the occurrence of postpartum depression: Further evidence that lowered n-PUFAs are related to major depression. Life Sci 2003; 73 (25) 3181-7.
29. Makrides M, Crowther CA, Gibson RA, Gibson RS, Skeaff CM. Docosahexaenoic acid and post-partum depression - is there a link?. Asia Pac J Clin Nutr 2003; 12 Suppl: S37.

30. Chiu CC, Huang SY, Shen WW, Su KP. Omega-3 fatty acids for depression in pregnancy. Am J Psychiatry 2003; 160(2): 385. Letter to the Editor. 